\title{
A new program for the hydraulic calculation of steam-water mixture pipelines in geothermal fields
}

\author{
Aleksandr Shulyupin ${ }^{1 *}$, Alla Chermoshentseva ${ }^{2}$ and Natalia Varlamova ${ }^{1}$ \\ ${ }^{1}$ Mining Institute FEB RAS, 51 Turgenev st., Khabarovsk, 680000, Russia; \\ ${ }^{2}$ Kamchatka State Technical University ,683003 Petropavlovsk-Kamchatsky, Russia
}

\begin{abstract}
A new mathematical model of steam-water flow in a pipeline, which takes into account the gravitational effect and satisfies new challenges in the development of geothermal fields with two-phase transportation of the heat carrier is presented. Taking into account the gravitational effect in the upstream flows, a method based on the "drift model", which determines the steam velocity averaged over the crosssection was used. A similar method is proposed for downstream flows, but it determines the water velocity averaged over the cross-section. In this case, analogs of empirical coefficients were used. These coefficients determined from the condition of equality of parameters calculated using different approaches in a horizontal flow. The model was verified, which showed positive results. The proposed model significantly expands the possibilities of hydraulic calculation of steam-water mixture pipelines in geothermal fields.
\end{abstract}

\section{Introduction}

The use of geothermal resources for the needs of the electric power industry is a promising, actively developing area, the development of which goes beyond the framework of subsidized projects. Recently, the development of geothermal resources is increasingly carried out on a commercial basis. In this regard, along with new promising studies, for example, in the field of creating enhanced geothermal systems [1, 2] and extracting geothermal energy without rise of underground fluids to the surface [3, 4], increased attention has been paid to the efficiency of using existing technologies.

The basis of modern geothermal electric power engineering is the development of hydrothermal resources associated with the rise to the surface of the heat carrier as a steamwater mixture. The tightening of environmental requirements, the widespread introduction of injection technologies and the development of technology for using the energy potential of the liquid phase in two-circuit systems have made it necessary to transport steam and water to a common place of use. In this case, it is advisable not to separate steam and water near the wellhead, but to transport the steam-water mixture through pipelines [5].

\footnotetext{
*Corresponding author: ans714@mail.ru
} 
The technology of two-phase transportation of the heat carrier began to be introduced at the end of the last century [6], and is actively used now [7]. In particular, two-phase transportation is the main one at the Mutnovsky field (Kamchatka), which generates about $90 \%$ of all domestic electricity from geothermal resources.

The steam-water mixture pipeline operation revealed the main problem - the choice of the optimal pipeline diameter. The small diameter is associated with large pressure losses during transportation, which leads to the need to increase the wellhead pressure of production wells or reduce the pressure at the power plant entrance, and this reduces the energy potential of the produced heat carrier. The large diameter is associated with significant financial costs of the pipeline construction, and also increases the risk of unstable operating modes of the heat carrier production and transportation system, which often make practical use of wells impossible.

This paper presents a new program for hydraulic calculation of steam-water mixture pipelines that corresponds modern challenges in the development of geothermal fields [8].

\section{Mathematical model of steam-water flow in a pipeline}

The mechanism of occurrence and development of macroscopic flow instability is considered in detail in $[9,10]$. On the basis of the theoretical provisions of these works, the proposed model uses the condition

$$
\frac{\partial \Delta p}{\partial G}>0
$$

where $\Delta p$ is the internal pressure drop in pipeline.

There are very few practical recommendations for the hydraulic calculation of steamwater geothermal flows in pipelines. The calculation of pipelines during the development of domestic fields was carried out using the MODEL computer program. There were no complaints about the discrepancy between the calculated and practical data (the operation of the pipelines was carried out in the range of nominal flow rates recommended by the program). The mathematical model underlying this program is designed for a narrow range of flow rates velocities and does not take into account the pressure drop gravitational component, which can cause violation of condition (1). In addition, the fact that this model does not meet modern challenges was noted in [8]. This indicates the need to develop a new mathematical model.

Internal pressure drop in the pipeline is expressed in terms of the components for friction, acceleration, gravity and local resistance

$$
\Delta p=\Delta p_{f}+\Delta p_{m}+\Delta p_{a}+\Delta p_{g}
$$

where $\Delta p$ is the total internal pressure drop in the pipeline, $\Delta p_{f}, \Delta p_{m}, \Delta p_{a}, \Delta p_{g}$ are the components of friction, local resistances, acceleration and gravity.

Recommendations for the practical determination of the individual components of formula (2) for two-phase medium depend on the parameters of the medium and the conditions of its transportation. The steam-water heat carrier transported through pipelines in geothermal fields is characterized by vapor phase in volume units; pipelines have a large diameter (from $0.3 \mathrm{~m}$ ). In a saturated environment, temperature is related to pressure. To maintain the temperature, which determines the energy potential of the heat carrier, during the transportation of the mixture, pressure losses are sought to be minimized, that is, as a rule, the pressure drops are small, and the composition of the mixture at the beginning and 
at the end of the pipeline differs little. Such conditions make it possible to neglect the component of the pressure drop for acceleration.

The friction component is determined by the formula obtained from the analysis of the forces acting on the selected section of the pipe

$$
d p_{f}=(4 \tau / D) d L
$$

where $\tau$ is the shear stress on the pipe wall, $D$ is the pipe diameter, $d L$ is the section length.

The total frictional pressure drop is determined by integrating (3) over the entire length of the pipeline, which is not difficult when the pipe diameter and shear stress can be considered constant. There are many recommendations for determining shear stress or direct frictional pressure drop in two-phase flows. These recommendations are validated experimentally and are applicable under certain experimental conditions. Due to the lack of experimental data, there are no special formulas for the conditions under consideration. A reasonable solution in this case seems to be a compilation of well-known formulas that showed good agreement with experimental data under conditions close to those considered.

The frictional pressure drop calculation within the MODEL program is focused on a narrow range of transportation velocities and is unacceptable for new challenges. To determine the shear stress in the new model, another formula is used. This formula is a compilation of known formulas: justified for well calculation [11] and the corresponding homogeneous model, which showed good results for large pipe diameters [7]

$$
\tau=\left(\rho_{l} v_{l}^{2}(1-\alpha)+\rho_{g} v_{g}^{2} \alpha+\rho_{w} w^{2}\right) \lambda / 16,
$$

where $\rho_{l}$ and $\rho_{g}$ are the densities of water and steam, $v_{l}$ and $v_{g}$ are the velocities of water and steam, $\alpha$ is the void fraction or true volumetric gas content (the ratio of the gas phase volume to the total volume of the considered element), $\lambda$ is the friction factor, $\rho_{w}$ and $w$ are the mixture density and velocity according to a homogeneous model.

To determine the pressure drop at the local resistance in the MODEL program, a formula, calculated for a wide range of velocities, was used. It makes it possible to use this formula in the new model

$$
\Delta p_{m}=0.7 \zeta \rho_{w} w^{2}
$$

where $\zeta$ is the local resistance coefficient for a single-phase flow.

The gravitational component of the pressure drop along the flow direction is determined by the formula

$$
d p_{g}=-\rho g \sin \theta d L
$$

where $\theta$ is the inclination angle of the pipe axis relative to horizontal plane, $\rho$ is the mixture density, determined by the formula

$$
\rho=\rho_{g} \alpha+\rho_{l}(1-\alpha)
$$

The sought value is determined by integration (6). The difficulty lies in determining the density of the mixture, which depends on the void fraction. A number of phenomena affecting the void fraction do not have a rigorous theoretical description; therefore, there is no universal theoretical formula based on the flow parameters for its determination. Practical problems are solved using formulas containing theoretically grounded correlations and empirically established coefficients. 
There are many formulas for determining the void fraction (in this particular case, volumetric steam content) $[12,13]$. In particular, there are correlations that claim to be universal, covering all conditions that took place in various experiments. However, there are no experimental data on the void fraction under the conditions of transportation of a steam-water geothermal mixture, at least for such an important parameter as pipe diameter. a diameter of $0.15 \mathrm{~m}$ is considered large in known experiments [14], while in this case, a diameter of geothermal pipelines more than $0.3 \mathrm{~m}$. In this situation, the only way to obtain the necessary formulas is to analyze the main determining factors using indirect experimental data. In this case, it is important to choose the general type of the sought correlations. The most theoretically justified is the type related to the drift model $[13,14]$. According to the drift model, the gas phase velocity averaged over the cross section is determined by the formula

$$
v_{g}=C_{0} w+v_{d},
$$

where $v_{g}$ is the gas phase velocity averaged over the cross section, $C_{0}$ is the distribution parameter, $w$ is the superficial mixture velocity equal to the mixture velocity of homogeneous model, $v_{d}$ is the drift velocity.

The drift characterizes the actual difference in phase velocities, and the distribution parameter takes into account the uneven distribution of phases in an uneven velocity field, which, as a result of averaging over the cross section, leads to a difference in the phase velocities even if their actual local values are equal. The drift velocity is determined by the influence of two factors: gravity, which tends to give the liquid phase an additional downward velocity, relative to the gas; hydrodynamic forces tending to impart larger velocity to the gas phase due to lower viscosity and inertia. Considering the second factor proportional to the mixture velocity, its influence can be taken into account within the distribution parameter. In this case, the value of $C_{0}$ in formula (8) is the sum of the distribution parameter and the correction due to the hydrodynamic part of the drift velocity, that is $C_{0}$ is a complex parameter. In this regard, in formula (8), it is sufficient to consider the influence of gravity for the drift velocity.

Based on the analysis of the factors influencing the difference in phase velocities due to gravity, and the analysis of ways to take these factors into account, the formula is proposed

$$
v_{d}=2.8(1-M) \sin \theta\left(g \sigma\left(\rho_{l}-\rho_{g}\right) / \rho_{l}^{2}\right)^{0.25},
$$

where $M$ is the Mach number, $\sigma$ is the surface tension coefficient.

In the same way, the formula of determine the distribution parameter is proposed

$$
C_{0}=1+k_{1}(1+\sin \theta+\cos \theta)
$$

where $k_{1}$ is upstream structure coefficient

$$
k_{1}=0.05(1-x)(1-M)\left(1-\rho_{g} / \rho_{l}\right),
$$

where $x$ is the vapor quality.

The vapor quality is related to the steam velocity

$$
\alpha=w_{g} / v_{g}
$$

where $w_{g}$ is the superficial steam velocity, determined as the ratio of the volumetric steam flow rate to the total cross section area of pipe. 
Formula (9) allows the possibility of a negative drift velocity value in downward flows. In this case, a drawback of the traditional drift model is revealed - the possibility of unrealistic values for the vapor velocity at a low superficial mixture velocity. The true steam velocity cannot take values lower than its superficial velocity, and the equality of these velocities is possible only at zero water flow rate, as well as at $v_{l} \rightarrow \infty$. To exclude this possibility, it is proposed to use a similar formula instead of (8) for downdrafts, but determining the water velocity

$$
v_{l}=K w-v_{d}
$$

where $v_{l}$ is the liquid phase velocity averaged over the cross section, $v_{d}$ is the drift velocity, which has a negative value for the downward flow in accordance with formula (9), $K$ is the analog of the distribution parameter

$$
K=1+k_{2}(1+\sin \theta+\cos \theta),
$$

where $k_{2}$ is the downward flow structure coefficient.

The choice of the formula for calculating the downstream structure coefficient was carried out in such a way that for the horizontal flow formulas (8) and (13) lead to the same result. It is easy to make sure that this is achieved when

$$
k_{2}=0.5\left(\frac{v_{l 0}}{w}-1\right),
$$

where $v_{l 0}$ is the water velocity for horizontal flow, determined by the traditional drift model based on formula (8).

Taking into account (13) - (15) and having determined the water velocity, the void fraction can be found

$$
\alpha=1-w_{l} / v_{l},
$$

where $w_{l}$ is the superficial water velocity defined as the ratio of the volumetric flow rate of water to the total sectional area of the pipe.

Formulas (2-16), as well as the stability criterion (1), are the basis of the proposed model for calculating the steam-water flow in the steam-water geothermal mixture surface pipelines.

The presented model was implemented in the SWIP-S program - the simplest version, when all values and their gradients were determined for one nodal point and were considered unchanged over the entire calculated interval. A similar approach was used when creating the MODEL program, which did not cause significant difficulties in practice; it is always possible to divide the pipeline into sections within which this simplification is acceptable.

\section{Program verification}

There are very few experimental data for verifying the developed program. Data for detailed verification, for example, for determining the void fraction, under the appropriate conditions are not available at all. At the same time, there is a wealth of experience in using the MODEL program for calculating pipelines at the Pauzhetsky and, especially, at the Mutnovsky fields. At the Mutnovsky field, in addition to much more experience in the application of this program, better monitoring of the flow characteristics of wells is carried out, which increases the reliability of judgments about the agreement of calculated and field 
data. In this regard, to verify the SWIP-S program, it is advisable to use a comparison with the calculation according to the MODEL program under typical conditions of its application at the Mutnovsky field: the internal diameter of the pipeline is $0.4 \mathrm{~m}$, the enthalpy of the mixture is $1200 \mathrm{~kJ} / \mathrm{kg}$, the pressure at the wellhead is $7.5 \mathrm{bar}$, the superficial velocity is $25-30 \mathrm{~m} / \mathrm{s}$, which corresponds to a flow rate of $50-60 \mathrm{~kg} / \mathrm{s}$ for the specified conditions. Since the MODEL program does not take into account the gravitational component, and local resistances are determined in the same way as in the new model, the verification was carried out for a horizontal pipe without the presence of local resistances. The pipe length was taken to be $100 \mathrm{~m}$. The calculated pressure drops are shown in Figure 1. In the nominal flow rate range, there is a good agreement.

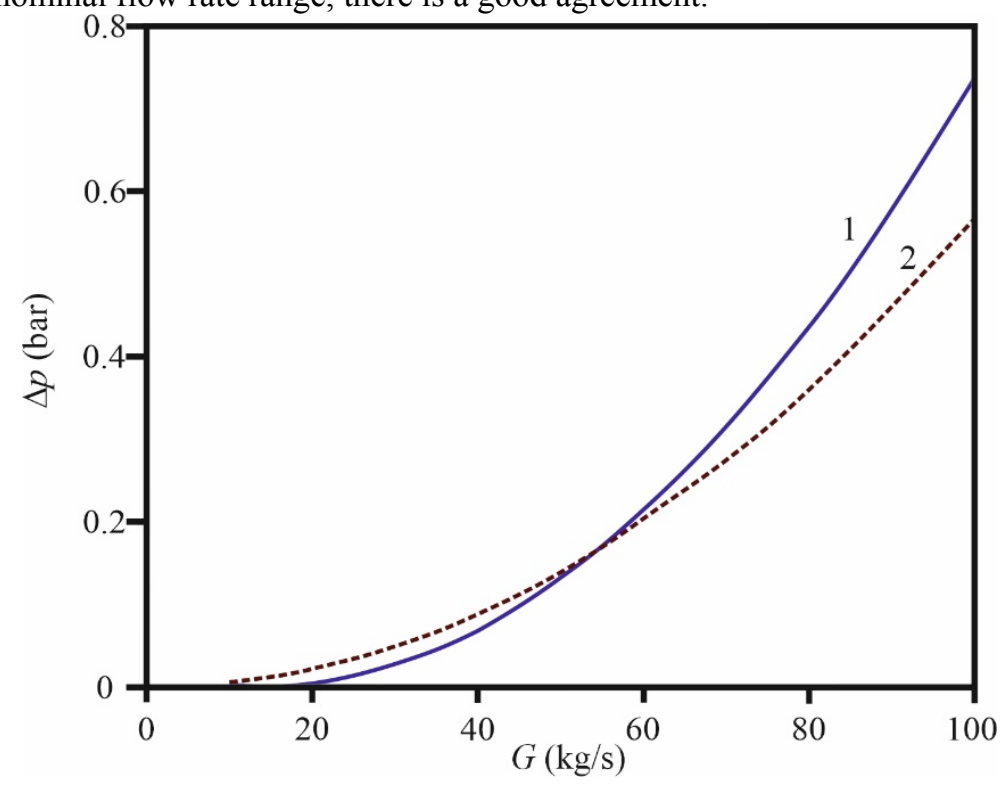

Fig. 1. A pressure drop calculated: 1 - MODEL program, 2 - SWIP-S program.

To verify the new program in inclined pipe conditions, the pipeline data from the Geo-1 well in the Mutnovsky field was used. This pipeline has the largest height difference of the steam-water mixture pipelines in operation in Kamchatka. Data on the pipeline: length 1050 $\mathrm{m}$, height difference from the beginning to the end $110 \mathrm{~m}$, inner diameter $0.406 \mathrm{~m}$, total coefficient of local resistance 8 . For verification, data obtained at two points in time: 16.09.2011 and 11.09.2019 were used. The parameters at the pipeline entrance and the calculation results are shown in the Table 1.

Table 1. The parameters at the pipeline entrance and the calculation results

\begin{tabular}{|c|c|c|c|c|c|}
\hline Date & $\begin{array}{c}\text { Flow rate, } \\
\mathrm{kg} / \mathrm{s}\end{array}$ & $\begin{array}{c}\text { Enthalpy, } \\
\mathrm{kJ} / \mathrm{kg}\end{array}$ & $\begin{array}{c}\text { Wellhead } \\
\text { pressure, bar }\end{array}$ & $\begin{array}{c}\text { Pressure drop } \\
\text { calculated, bar }\end{array}$ & $\begin{array}{c}\text { Pressure } \\
\text { drop testing, } \\
\text { bar }\end{array}$ \\
\hline 16.09 .2011 & 65.0 & 1221 & 11.3 & 1.52 & 1.5 \\
\hline 11.09 .2019 & 65.3 & 1121 & 8.9 & 1.76 & 1.8 \\
\hline
\end{tabular}

When calculating the total pressure drop, the pipeline was divided into 7 equal sections. The measured differential pressure has an error of \pm 0.2 bar. Despite the introduction of approximate values for some coefficients into the model, the table shows good agreement between the calculated and measured pressure drops.

When designing this pipeline, the MODEL program was used. Taking into account the absence of upstream sections, in order to reduce hydraulic losses, the choice of the diameter 
was carried out according to the maximum value corresponding to the condition of applicability of the specified program. It is important to note that on 16.09.2011, the diameter at the beginning of the pipeline exceeded the maximum value recommended by the MODEL program $(0.401 \mathrm{~m})$. In such conditions, the calculation of the pressure drop for friction using the MODEL program should give underestimated values. In reality, the pressure drop for friction and local resistance, calculated using the MODEL program, was 1.48 bar. Taking into account the gravitational component even with a low density of the mixture, corresponding to the homogeneous model, the total pressure drop is 1.22 bar, which is significantly less than the measured value.

\section{Conclusions}

Thus, the calculation using the SWIP-S program under typical conditions for transporting a steam-water geothermal mixture at the Mutnovsky field is in good agreement with the calculation using the MODEL program, which, under these conditions, is in good agreement with the experimental data. For atypical conditions (significant pipeline slope, low flow rates), the new program looks preferable.

A detailed study of the features of the application of the new program in various conditions of transportation of a steam-water geothermal coolant, including different geometry of the routes, and the accumulation of data in comparison of the calculated and experimental results will allow developing a methodology for the most rational application of this program, as well as identifying possible directions for its improvement.

The reported study was funded by RFBR, project number 20-05-00161.

\section{References}

1. J.H. Norbeck, M.W. McClure, R.N. Horne, Geothermics, 74 (2018)

2. J. Zhang, J. Xie, X. Liu, International Journal of Heat and Mass Transfer, 134 (2019)

3. T. Renaud, P. Verdin, G. Falcone, International Journal of Heat and Mass Transfer, 143 (2019)

4. S. Iry, R. Rafee, Geothermics, 77 (2019)

5. J.H. Smith, Geothermal energy: review of research and development, Earth sciences (UNESCO, Paris, 1973)

6. K.C. Lee, D.G. Jenks, Proceedings, 11-th New Zealand Geothermal Workshop (1989)

7. Rizaldy, S.J. Zarrouk, Proceedings 38th New Zealand Geothermal Workshop (2016)

8. A. Shulyupin, N. Varlamova, E3S Web of Conferences, 56, 01008 (2018)

9. A.N. Shulyupin, Stability of operate mode of steam-water well (Amurprint, Khabarivsk, 2018)

10. A.N. Shulyupin, International Journal of Heat and Mass Transfer, 105 (2017)

11. A.N. Shulyupin, A.A. Chermoshentseva, Mathematical Models and Computer Simulations, 9, 1 (2017)

12. M.A. Woldesemayat, A.J. Ghajar, International Journal of Multiphase Flow, 33 (2007)

13. Y. Xu, X. Fang, Applied Thermal Engineering, 64, 242-251 (2014)

14. Z Dang., Z. Yang, X. Yang, M. Ishii, International Journal of Multiphase Flow, 111 (2019) 